\title{
Analysis of the adhesion activity of peritoneal macrophages after exposure to radiation from a gas-discharge plasma and mercury lamp
}

\author{
Evgenia Arkhipova, Igor Piskarev and Irina Ivanova \\ Central Research Laboratory, Privolzhsky Research Medical University, Nizhny Novgorod, Russia
}

\begin{abstract}
The aim of the work was to study the influence of UV radiation of a spark discharge plasma and a mercury lamp on the state of membrane structures of peritoneal macrophages. The objects of the study were peritoneal macrophages of rats. The total number of cells after exposure and their viability were analyzed. Oxidative modification of proteins was recorded by fluorescence of tryptophan, tyrosine and products of non-enzymatic glycosylation of proteins. The concentration of sialic acids was determined spectrophotometrically, and the intensity of adhesion properties of cells was estimated by the ability to adhere to the plastic. It was shown that the radiation of a spark discharge plasma and UV lamp with the selected exposure regimes affect the structural components of membranes of peritoneal macrophages. The ability to adhere is enhanced by short exposure regimes, and under long-term conditions, adhesion properties decrease. The change in adhesion is probably associated with a decrease in the concentration of sialic acids on the cell surface, as well as with the intensification of oxidative modification of proteins. It has been established that spark plasma and UV lamp radiation promote the oxidation of aromatic amino acids and the accumulation of glycosylation products of proteins.
\end{abstract}

Key words: Peritoneal macrophages — Gas-discharge plasma - UV radiation — Fluorescence of amino acids - Adhesion

\section{Introduction}

Cells of the mononuclear phagocyte system are of great importance in maintaining the body's resistance. The intensity of the immune response and the body's homeostasis depend on the functional activity of phagocytes (Phan et al. 2017), and the integrity of the macrophage membrane and its structural organisation affect intracellular processes and various metabolic reactions (Köberlin et al. 2016). The study of physicochemical factors that are able to increase phagocytic activity in infectious or neoplastic processes is a topical area of research; the biological effects of cold gasdischarge plasma and plasma radiation have recently been actively studied (Sasai et al. 2010; Kubinova et al. 2017). In the process of generating gas discharge plasma radiation, and

Correspondence to: Evgenia Arkhipova, Central Research Laboratory, Privolzhsky Research Medical University, Minin and Pozharsky Sq., 10/1, Nizhny Novgorod, 603005, Russia

E-mail: arhipova@nnovgorod.ru particularly in the UV range, radical products are formed that participate in oxidation and reduction reactions, and these are capable of modifying macromolecules and metabolic processes in cells in various ways (Kanazawa et al. 2011; Piskarev et al. 2016a).

The cytotoxic and bactericidal activation of mononuclear cells after exposure to gas-discharge plasma has previously been demonstrated (Ivanova and Zaslavskaya 2009; Arkhipova and Ivanova 2013; Astafyeva and Ivanova 2017), and the immunomodulating properties of radiation, lasers and pulsed UV radiation are known. The observed effects depend on the radiation dose (Luza and Hubacek 1996; Klebanov et al. 1998; Dube et al. 2003; Salmin et al. 2014), although the effects of physical factors on the structural components of the cell membranes determining the adhesive activity have not been sufficiently studied.

An examination of the influence of UV radiation from different sources and their comparative analysis will therefore bring us closer to an understanding of the mechanisms of macrophage activation. In view of this, the purpose of 
this work is to study the effects of gas-discharge plasma radiation and mercury lamp UV radiation on the state of the membrane structures of peritoneal macrophages.

\section{Materials and Methods}

The object of the study was peritoneal macrophages in Wistar rats. The experiments were performed in vitro. To obtain a suspension of macrophages, animals (males weighing 200-220 g) were intraperitoneally injected with a sterile saline solution, and the animals were decapitated after 20 minutes under isoflurane anaesthesia.

In terms of ethical approval, all procedures complied with the Directive of 22nd September 2010 (2010/63/EU) of the European Parliament and the Council of the European Union, and were approved by the local ethical committee of Nizhny Novgorod State Medical Academy, Nizhny Novgorod (Russia) (protocol No. 2, 8th February 2016). All efforts were made to minimise the number of animals used and their suffering.

UV radiation was generated using a DKB9 mercury lamp (Russia). The spectrum of the DKB9 lamp was monochromatic, with $\lambda=253.7 \mathrm{~nm}$; its continuous power was $9 \mathrm{~W}$, and the mean photon flux was $5.4 \times 10^{-8} \mathrm{~mol}\left(\mathrm{~cm}^{2} \mathrm{~s}\right)^{-1}$. Gas-discharge plasma radiation was generated using a PILIMINIR-10 series experimental device (Skobeltsin Institute of Nuclear Physics, Lomonosov Moscow State University, Russia) with the following parameters: pulse duration $100 \mathrm{~ms}$, high-voltage

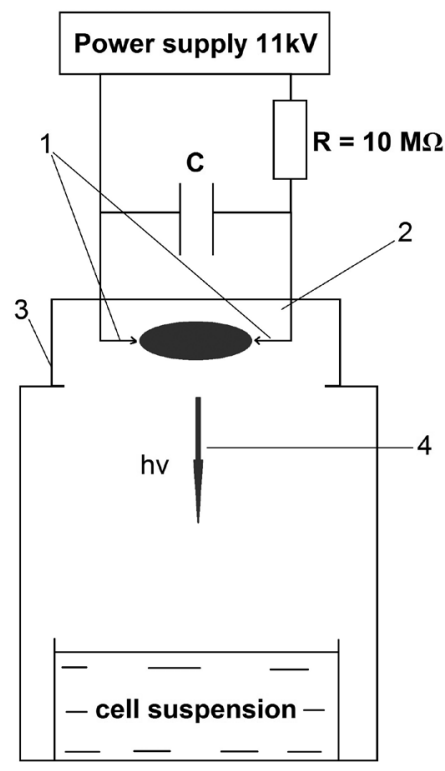

Figure 1. Experimental setup of Pilimin IR-10 series source for the generation of spark discharge gas plasma radiation. C, capacitor; 1 , electrodes; 2 , spark arrester; 3 , arrester case; 4 , radiation plasma. power supply $11 \mathrm{kV}$, pulse capacitor value $3.3 \mathrm{nF}$, pulse energy $5.9 \times 10^{-2} \mathrm{~J}$, repetition frequency $10 \mathrm{~Hz}$. The radiation spectrum was continuous in the range $200-360 \mathrm{~nm}$ with a maximum at $\lambda=220 \mathrm{~nm}$, and a mean photon flux of ( 1.26 $\pm 0.2) \times 10^{-10} \mathrm{~mol}\left(\mathrm{~cm}^{2} \mathrm{~s}\right)^{-1}$ (Fig. 1). Suspensions of peritoneal macrophages were treated non-invasively at a distance of $3 \mathrm{~cm}$ for $30,60,300,600$ and $1200 \mathrm{~s}$. Cells that had not been exposed to radiation were used as controls.

The total number of cells after exposure was counted using a Scepter automatic cytometer (Millipore, USA). The viability of the cells was determined using the colorimetric method, by reaction with water-soluble blue $3-(4,5-\mathrm{Di}$ methylthiazol-2-yl)-2,5-diphenyl tetrazolium bromide (MTT test). The oxidative modification of proteins was monitored via the fluorescence of tryptophan and tyrosine residues, and by the products of non-fermentative protein glycosylation. Tyrosine fluorescence was measured using $\lambda_{\text {excit }}=275 \mathrm{~nm}$ and $\lambda_{\text {emiss }}=303 \mathrm{~nm}$, while tryptophan fluorescence was studied at $\lambda_{\text {excit }}=288 \mathrm{~nm}$ and $\lambda_{\text {emiss }}=350 \mathrm{~nm}$ (Piskarev et al. 2016b). The glycosylated protein content was determined using a fluorometric method, with $\lambda_{\text {excit }}=$ $370 \mathrm{~nm}$ and $\lambda_{\text {emiss }}=421 \mathrm{~nm}$ (Munch et al.1997). Fluorescence spectra were recorded using a Fluorat 02 Panorama spectrofluorimeter (Lumex, Russia). The concentration of sialic acids was determined spectrophotometrically using a SIALOTEST-80T reagent kit (NPC EKO Service, Russia). To assess the intensity of cell adhesion to plastic surfaces, peritoneal macrophages were introduced into a Petri dish (of diameter $40 \mathrm{~mm}$ ) together with a DMEM culture medium (PanEko, Russia) in the ratio 1:3 and incubated for 60 minutes at $37^{\circ} \mathrm{C}$. The preparations were observed microscopically at 600 -fold magnification, and the attached cells were counted in ten fields of vision.

The results were presented as mean \pm SEM. Statistical significance was determined using the Mann-Whitney test. Two sets of experimental data were considered to belong to different populations when $p<0.05$.

\section{Results and Discussion}

The total number of cells after each treatment was counted to evaluate the cytotoxic effect of UV radiation from the spark discharge plasma device and the mercury lamp (Fig. 2). The numbers of peritoneal macrophages decreased by $20.4 \%$ and $32.3 \%$ after exposure to spark discharge plasma radiation for exposure times of 600 and $1200 \mathrm{~s}$, respectively, compared with the control group. On exposure to UV light from a mercury lamp, the total cell numbers decreased by $19 \%, 24 \%$ and $43 \%$ after exposure times of 300, 600 and 1200 seconds, respectively. This reduction in total cell numbers after exposure to UV radiation from the spark discharge device and from the mercury lamp indicates that both types of radiation 


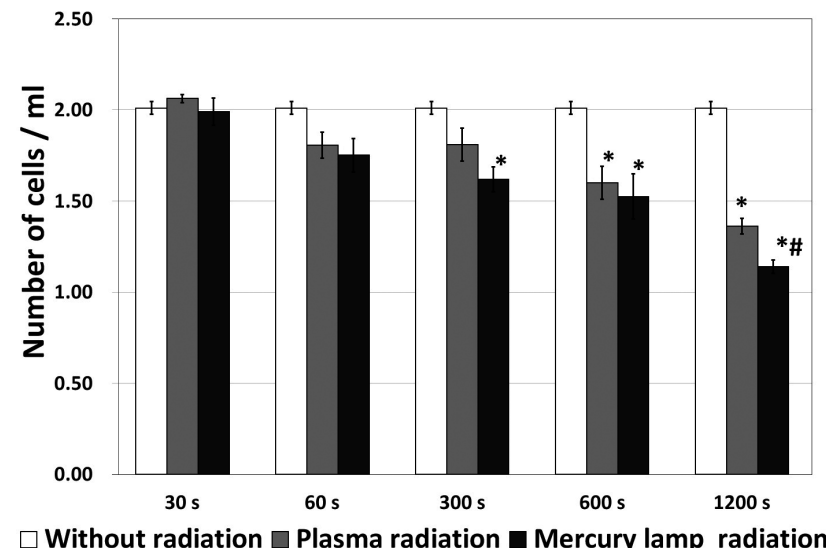

Figure 2. Total number of peritoneal macrophages after exposure to UV spark discharge plasma and mercury lamp radiation. ${ }^{\star} p<0.05$ in comparison with the control group; \# $p<0.05$ in comparison with the plasma radiation group.

have a cytotoxic effect. In this experiment, peritoneal macrophages were more resistant to spark discharge radiation.

The number of cells began to decrease after $300 \mathrm{~s}$ of exposure to the mercury lamp radiation; at the maximum exposure time, the decrease in the total number of cells was $16 \%$ greater than that for exposure to plasma radiation. In addition, the viability of the cells was studied in order to assess the effect of the radiation on the state of the peritoneal macrophages. The viability of the population of peritoneal macrophages after exposure to UV radiation from a spark discharge of durations of 300, 600 and 1200 s decreased by $14.6 \%, 49.7 \%$ and $55.6 \%$, respectively (Fig. 3 ). When the mercury lamp was used, the viability of the peritoneal macrophages also decreased after exposure for durations of 300 , 600 and 1200 s by $15.4 \%, 55.75 \%$ and $63.8 \%$, respectively. It is therefore shown that long-term exposure regimes of 600 and $1200 \mathrm{~s}$ have a cytotoxic effect on peritoneal macrophages, and that these not only reduce the total number of cells but also affect the viability.

It is possible that the cytotoxic effect of UV radiation from spark discharge plasma and mercury lamp radiation is associated with the chemical modification of membrane structures as a result of redox reactions, since it is known that radiation from spark discharge and mercury lamps are sources of active products such as nitrogen oxides, free radicals and ammonium ions (Piskarev 2016). The more pronounced cytotoxic effect of UV radiation from a mercury lamp can be attributed firstly to the monochromatic spectrum of the radiation from the mercury lamp, and secondly to the fact that the photon flux of the mercury lamp is 400 times greater; it is also known that the yield of ammonium ions $\left(\mathrm{NH}_{4}{ }^{+}\right)$ under UV mercury lamp radiation is 100 times greater than for spark plasma radiation (Piskarev et al. 2016b).
$\mathrm{NH}_{4}{ }^{+}$ions can cause depolarisation of the cell membrane, disrupt $\mathrm{Na}^{+} \mathrm{K}^{+}$ATPase activity due to competition of $\mathrm{NH}_{4}{ }^{+}$ with $\mathrm{K}^{+}$ions, promote the development of oxidative-nitrosative stress and lead to the formation of active metabolites of oxygen and nitrogen (Martinelle and Haggstrom 1993; Kosenko et al. 1997; Dai et al. 2013; Skowronska and Albrecht 2013). Since various active particles are generated under the influence of UV radiation from spark discharge plasma and a mercury lamp, we can assume that these can initiate a modification of the lipid and protein components of the membranes of peritoneal macrophages, leading to a change in structure. In previous studies, we showed that UV radiation from spark discharge plasma and a mercury lamp leads to a change in the lipid spectrum of peritoneal macrophages, but that lipoperoxidation processes were not activated (Arkhipova and Ivanova 2016, 2017).

It is known that in a state of oxidative stress, attacks from reactive oxygen species primarily affect proteins rather than lipids. Various enzymes, ion channels and ATPases are proteins in nature, and are responsible for many functional processes in the cells, and the processes of oxidative modification of proteins therefore play a leading role in the violation of the functional activity of the cell membrane. Thus the oxidative modification of proteins is one of the earliest events in, and provides the most sensitive test for, the oxidative modification of macromolecules (Witko-Sarsat et al. 1996). In this case, the oxidative modification of proteins can be associated not only with the breakdown of the structure of the polypeptide chain, but also with individual amino acid residues, leading to a change in the conformation of proteins and their regulatory and functional properties (Ansary and Rshid 2010; Abalenihina and Fomina 2016). Hence, the fluorescence of tryptophan, tyrosine and the glycosylated proteins in peritoneal macrophages was evaluated in order to estimate the intensity of oxidative processes.

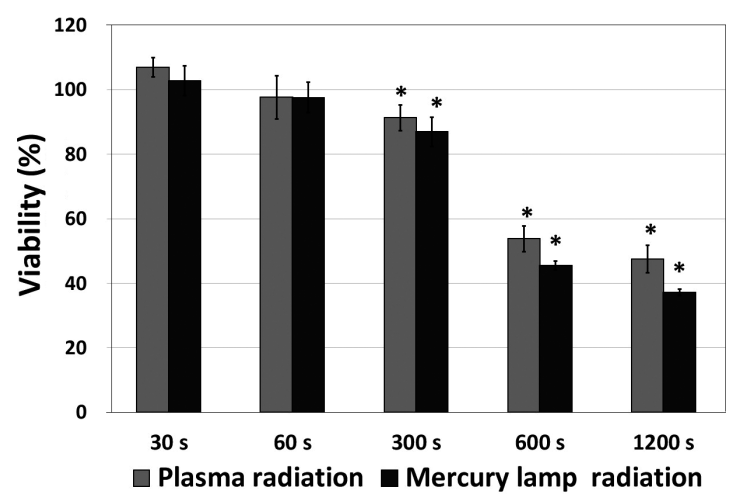

Figure 3. Viability of peritoneal macrophages after UV irradiation by a plasma generator and mercury lamp. ${ }^{*} p<0.05$ in comparison with the group irradiated for 30 seconds. 


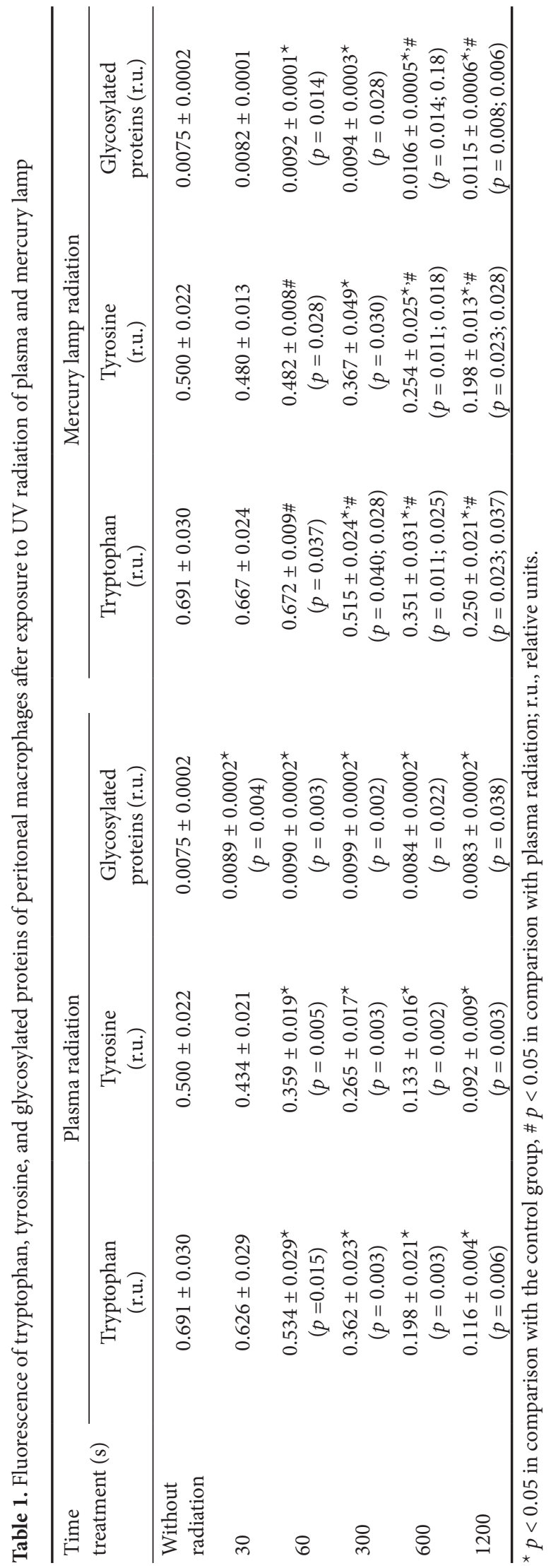

UV radiation from spark plasma discharge, processed for $60,300,600$ and $1200 \mathrm{~s}$, led to gradual decreases in the fluorescence of tryptophan and tyrosine by $22-83 \%$ and $28-81 \%$ respectively, as compared with the control group (Table 1). A $12-32 \%$ increase in glycosylated proteins was detected for all periods of exposure to radiation from spark discharge plasma, but no time dependence could be established. The oxidation of tyrosine and tryptophan residues is accompanied by decreased fluorescence, which is characteristic for tyrosine and tryptophan, but the accumulation of glycosylated protein products is accompanied by an increase in fluorescence.

UV irradiation from a mercury lamp for 300, 600 and $1200 \mathrm{~s}$ also led to a gradual decrease in the fluorescence of tryptophan and tyrosine of $25-63 \%$ and $26-60 \%$, respectively. The exposure of peritoneal macrophages to mercury lamp radiation for periods of $60,300,600$ and $1200 \mathrm{~s}$ led to a gradual accumulation of glycosylated protein products, with increases of $22-53 \%$ compared with the control group. Thus, intensified oxidative protein modification was seen in peritoneal macrophages following UV irradiation by the spark discharge plasma device and the mercury lamp, as evidenced by statistically significant increases in the content of glycosylated proteins and the decreased fluorescence of tyrosine and tryptophan residues.

The decreased tyrosine and tryptophan fluorescence may indicate a change in the conformation of protein molecules due to the unfolding globule amino acid residues becoming more accessible for oxidants (Giulivi et al. 2003; Hixon and Reshetnyak 2009). A change in the conformation of protein molecules can lead to changes in their functional and regulatory capabilities. Protein glycosylation is a nonenzymatic covalent reaction between the free amino groups of the protein molecule and glucose. An increased glycosylation level can lead to changes in intracellular and intercellular signalling and changes in the structural state of cell membranes, and may disturb the receptor apparatus. It can thus affect the functional activity of peritoneal macrophages (Yim et al. 2000; Ansary and Rshid 2010). In addition, enhancement of the oxidative modification of proteins is possible, and is associated with an increase in the microviscosity of the membranes of peritoneal macrophages, as shown in a previous study (Arkhipova and Ivanova 2017); this changes the degree of immersion of proteins in the lipid bilayer, and may aggregate membrane proteins with the formation of intra- and intermolecular cross-links (Artsruni et al. 2013).

Spark discharge plasma radiation promoted a more intensive oxidation of tyrosine and tryptophan, with increases of $25-53 \%$ and $20-53 \%$ respectively compared with mercury lamp radiation, and the accumulation of the products of protein glycosylation was slower by $20-28 \%$. It is known that the residues of the amino acids tyrosine and tryptophan 


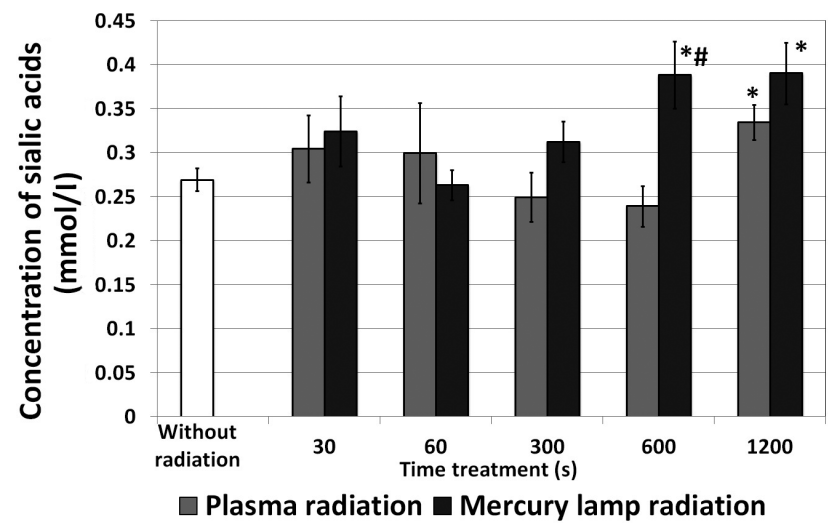

Figure 4. Concentration of sialic acids in the extracellular space after exposure to UV plasma and mercury lamp radiation. ${ }^{\star} p<0.05$ in comparison with the control group; \# $p<0.05$ in comparison with the plasma radiation group.

are most sensitive to damage under oxidative stress conditions, due to the system of developed double bonds in their structures (Abalenihina and Fomina 2016). It is likely that the radical products of spark plasma discharge radiation act primarily on the double bonds of aromatic amino acids. In order to clarify the mechanisms of protein glycosylation under UV radiation from a mercury lamp and modification of amino acids under spark plasma radiation, more detailed physicochemical investigations are needed.

The obtained results indicate that under the influence of UV radiation from plasma and a mercury lamp, oxidation processes are activated and reorganisation of the membrane structure occurs. Thus, in order to assess the state of the surface membrane structures of peritoneal macrophages, we studied the process of desialysation based on the concentration of sialic acids in the intercellular space. Sialic acids, which are derivatives of neuraminic acid, are components of glycoproteins and glycolipids in the plasma membranes of peritoneal macrophages (Kelm and Schauer 1997). Located at the end of the carbohydrate side chains of membranes, they determine the physicochemical properties of cells, and take part in intercellular interactions, migration, adhesion and macrophage polarisation (Kelm and Schauer 1997; Seyrantepe et al. 2010).

After treatment of the cell suspension with UV radiation from spark discharge plasma, a $24 \%$ increase in the level of sialic acids was found after $1200 \mathrm{~s}$ (Fig. 4). Following exposure to UV radiation from a mercury lamp for 600 and $1200 \mathrm{~s}$, the levels of sialic acids increased by $44.2 \%$ and $45 \%$, respectively. The decrease in the concentration of sialic acids on the surface of the membrane reflects the processes of desialysation, which can lead firstly to a violation of the conformation of receptors and their ability to interact, and secondly to a decrease in charge and a decrease in the ability of cells to repulse one another (Goswami and Koner 2002; Seyrantepe et al. 2010).

It is possible that the UV radiation from the spark plasma and the mercury lamp affects the activation of the neuraminidase enzyme, since it participates in the desialysation and sialing processes; by changing the qualitative and quantitative composition of glycoproteins on the cell membranes, this enzyme modulates the cell response to certain signals (Varki and Gagneux 2012, Wang et al. 2016). In addition, the concentration of sialic acids on the cell membrane can also be affected by the free radicals that accumulate in the liquid during the generation of UV radiation from the spark plasma and mercury lamp. This is consistent with our data on the fluorescence of tyrosine residues. In prior work (Goswami and Koner 2002), a correlation was found between desialysation and the accumulation of carbonyl derivatives of proteins under oxidative stress conditions. This is consistent with our data on the fluorescence of tyrosine and tryptophan residues and the accumulation of glycosylated proteins.

Changes in the surface charge as a result of desialysation can affect the functional properties of cells, and in particular the ability to adhere. The adhesion ability of peritoneal macrophages is an important property for the full implementation of their immune function, including phagocytic activity (Adamenko 2000). An adhesion test also allows us to evaluate the state of the receptor apparatus of cells. Exposure to spark discharge plasma UV radiation for 30 and $60 \mathrm{~s}$ resulted in increases in the number of cells attached to plastic Petri dishes by $50 \%$ and $25 \%$ respectively (Fig. 5). After long exposure times of 600 and $1200 \mathrm{~s}$, there was a decrease in the number of cells attached to the plastic of $83 \%$ in each

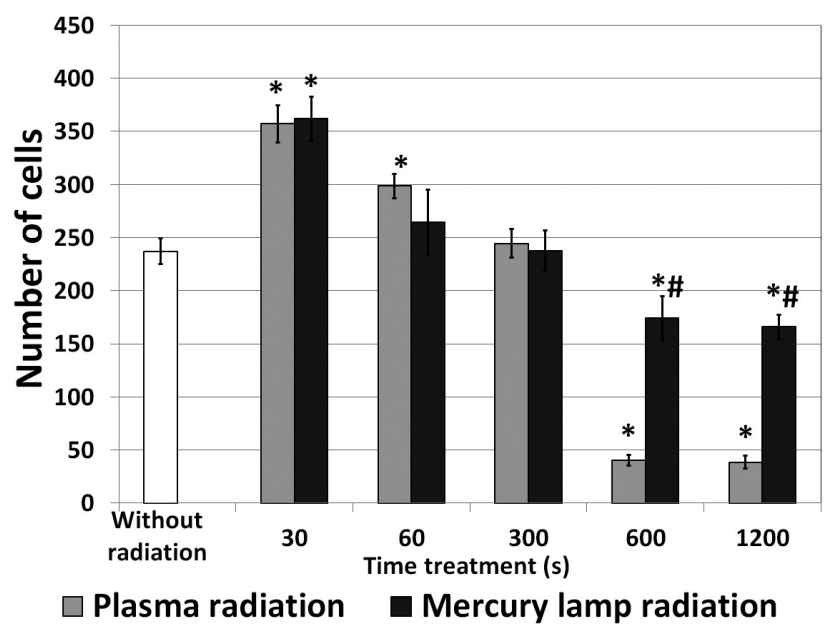

Figure 5. Increases and decreases in the adhesive ability of peritoneal macrophages after exposure to UV radiation from plasma and a mercury lamp. ${ }^{*} p<0.05$ in comparison with the control group; $\# p<0.05$ in comparison with the plasma radiation group. 
case. Mercury lamp UV radiation also changed the adhesive properties of macrophages. An increase in the number of attached cells by $52 \%$ after exposure for 30 seconds was found. After exposure for 600 and $1200 \mathrm{~s}$, decreases in the number of attached cells of $26 \%$ and $30 \%$ were also observed, compared with the controls.

An increase in the adhesion of phagocytic cells to the substrate is one of the factors indicating cell activation. Hyperadhesivity is accompanied by a reorganisation of cells, short-lived point contact, filopodia, and folds in the cellular margin, which improve contact with the substrate (Ciobanasu et al. 2012). Specific molecules of the integrin family are involved in the stabilisation of adhesion contacts, and these are contained in the plasma membrane and cytoplasm; they are derived on the surface only as a result of additional stimulation (Sanchez-Madrid and del Pozo 1999). Alterations in the cytoskeleton are also indicated by changes in the microviscosity properties of the membranes of peritoneal macrophages (Arkhipova and Ivanova 2017), since it has been shown in a number of studies that the activation of neutrophils increases the stiffness of the cell (Roca-Cusachs et al. 2006).

The reduction in the adhesive properties of peritoneal macrophages for long periods of exposure may be associated with a decrease in the expression of integrin receptors on the membrane surface, and thus with the loss of sialic acids and a change in the charge of the membranes; this is consistent with an increase in their concentration in the extracellular liquid. Hence, UV radiation from both the spark discharge plasma device and the mercury lamp affects the adhesive properties of peritoneal macrophages. For short exposure times of 30 and $60 \mathrm{~s}$, the ability to adhere increases, but long exposure periods of 600 and $1200 \mathrm{~s}$ lead to a decrease in the intensity of adhesion and an increase in the concentration of sialic acids in the extracellular region. The intensity of the adhesive activity of cells after exposure to UV radiation from spark discharge plasma is reduced by a factor of four compared to that seen after exposure to a mercury lamp. It is likely that under the action of spark discharge plasma radiation, the expression of integrin receptors may decrease and the structure of the integrin receptors subject to the action of active particles may be disrupted.

\section{Conclusion}

The results show that the studied UV radiation regimes of the spark discharge plasma and mercury lamp affect the state of the membrane structures of peritoneal macrophages. It has been established that after exposure to UV plasma radiation, the integrity of peritoneal macrophages persists to a greater extent $(16.2 \%)$ than after exposure to UV radiation from a mercury lamp, meaning that the membrane of the peritoneal macrophages is more resistant to radiation from spark discharge plasma. The viability of peritoneal macrophages after exposure to UV plasma radiation, although not statistically significant, is still greater (an increase of 8.2\%) than after exposure to UV radiation from a mercury lamp.

In addition, the UV radiation from spark discharge plasma promotes the more intensive oxidation of aromatic amino acids in comparison with that from a mercury lamp, with that of tyrosine increasing by $25.5-53.5 \%$ and that of tryptophan by $20.5-53.6 \%$. However, the accumulation of glycosylation products of proteins is $20.7-27.8 \%$ slower than when a mercury lamp is used.

The concentration of sialic acids in the extracellular space for a suspension of cells of peritoneal macrophages after treatment with UV radiation from a spark discharge plasma for the maximum duration ( $1200 \mathrm{~s}$ ) is $20.8 \%$ lower than after exposure to a mercury lamp.

The exposure to UV radiation for $30 \mathrm{~s}$ from both spark discharge plasma and a mercury lamp increases the adhesive activity of peritoneal macrophages by $50.5 \%-52.6 \%$. Exposure for 600 and $1200 \mathrm{~s}$ to UV radiation from spark discharge plasma decreases the adhesive activity of peritoneal macrophages by a factor of 4.35-4.31 compared with UV radiation from a mercury lamp.

These increases and decreases in the adhesive activity after exposure to the UV radiation of a spark discharge plasma undoubtedly require further study, and are likely to be useful both in reducing the immune responses of an organism and in autoimmune processes.

\section{References}

Abalenihina UV, Fomina MA (2016): The state of oxidative modification of tyrosine and tryptophan under in vivo modulation of nitric oxide synthesis. Eruditio Juvenium 1, 7 - 10

Adamenko GP (2000): The role of cell adhesion in receptor mechanisms of interaction of poly- and mononuclear phagocytes of human blood. J. Immunol. 1, 57

Ansary NA, Rshid Z (2010): Non-enzymatic glycation of proteins: from diabetes to cancer. Biomed. Khim. 56, 168-178 https://doi.org/10.18097/pbmc20105602168

Arkhipova EV, Ivanova IP (2013): The Effect of non-coherent impulse radiation on functional status of mononuclear cells in experiment. Sovremennye Tehnologii v Medicine 5, 27-31

Arkhipova EV, Ivanova IP (2016): Microviscosity and peroxide oxidation of lipids of peritoneal macrophages after exposure to UV radiation and radiation from gas-discharge plasma. Med. Akad. Z. 16, 46-47

Arkhipova EV, Ivanova IP (2017): Membrane structural condition and functional activity of peritoneal macrophages after gas discharge exposure. Sovremennye Tehnologii v Medicine 9, 55-63 https://doi.org/10.17691/stm2017.9.3.07 
Artsruni GG, Saakian GV, Pogosian GA (2013): The in vitro influence of the external electrostatic field on the physical parameters of erythrocyte membrane. Biofizika 25, 1022-1027 https://doi.org/10.1134/S0006350913060031

Astafyeva KA, Ivanova IP (2017): Analysis of cytotoxic effects of medical gas-discharge devices. Sovremennye tehnologii v medicine 9, 115-122 https://doi.org/10.17691/stm2017.9.1.15

Ciobanasu C, Faivre B, Le Clainche C (2012): Actin dynamics associated with focal adhesions. Int. J. Cell Biol. 2012, 1-9

Dai H, Song D, Xu J, Li B, Hertz L, Peng L (2013): Ammoniainduced $\mathrm{Na}, \mathrm{K}$-ATPase/ouabain-mediated EGF receptor transactivation, MAPK/ERK and PI3K/AKT signaling and ROS formation cause astrocyte swelling. Neurochem. Int. 2013 (Sep), 1-16 https://doi.org/10.1016/j.neuint.2013.09.005

Dube A, Bansal H, Gupta PK (2003): Modulation of macrophage structure and function by low level $\mathrm{He}-\mathrm{Ne}$ laser irradiation. Photochem. Photobiol. Sci. 2, 851-855

Giulivi C, Traaseth NJ, Davies KJ (2003): Tyrosine oxidation products: analysis and biological relevance. Amino Acids 25, $227-232$

https://doi.org/10.1007/s00726-003-0013-0

Goswami K, Koner BC (2002): Level of sialic acid residues in platelet proteins in diabetes, aging, and Hodgkins lymphoma: a potential role of free radicals in desialylation. Biochem. Biophys. Res. Commun. 297, 502-505 https://doi.org/10.1016/S0006-291X(02)02241-6

Hixon J, Reshetnyak YK (2009): Algorithm for the Analysis of Tryptophan Fluorescence Spectra and Their Correlation with Protein Structural Parameters. Algorithms 2, 1155-1176 https://doi.org/10.3390/a2031155

Ivanova IP, Zaslavskaya MI (2009): Biocydic effect of the spark discharge non-coherent impulse radiation in experiments in vitro and in vivo. Sovremennye Tehnologii v Medicine 1, 28-31

Kanazawa S, Kawano H, Watanabe S, Furuki T, Akamine S, Ichiki R, Ohkubo T, Kocik M, Mizeraczyk J (2011): Observation of $\mathrm{OH}$ radicals produced by pulsed discharges on the surface of a liquid. Plasma Sources Sci. Technol. 20, 383-391 https://doi.org/10.1088/0963-0252/20/3/034010

Kelm S, Schauer R (1997): Sialic acids in molecular and cellular interactions. Int. Rev. Cytol. 175, 137-240

Klebanov GI, Teselkin Y, Babenkova IV, Bashkujeva T, Chichuk TV, Vladimirov Y (1998): Low-power laser irradiation induces leukocyte priming. Gen. Physiol. Biophys. 17, 365-376

Kosenko E, Kaminsky Y, Kaminsky A, Valencia M, Lee L, Hermenegildo C, Felipo V (1997): Superoxide production and antioxidant enzymes in ammonia intoxication in rats. Free Radic. Res. 27, 637-644

Köberlin MS, Heinz LX, Superti-Furga G (2016): Functional crosstalk between membrane lipids and TLR biology. Curr. Opin. Cell Biol. 39, 28-36 https://doi.org/10.1016/j.ceb.2016.01.010

Kubinova S, Zaviskova K, Uherkova L, Zablotskii V, Churpita O, Lunov O, Dejneka A (2017): Non-thermal air plasma promotes the healing of acute skin wounds in rats. Scient. Rep. 7, 1-11 https://doi.org/10.1038/srep45183

Luza J, Hubacek J (1996): In vitro He-Ne laser effect on some immunological functions of the polymorphonuclears and monocytes in rabbits. Acta Univ. Palacki Olomuc Fac. Med. 140, 43-46

Martinelle K, Haggstrom L, (1993): Mechanisms of ammonia and ammonium ion toxicity in animal cells: transport across cell membranes. J. Biotechnol. 30, 339-350 https://doi.org/10.1016/0168-1656(93)90148-G

Munch G, Keis R, Wessels A (1997): Determination of advanced glycation endproducts in serum fluorescence spectroscopy and competitive ELISA. Eur. J. Clin. Chem. Clin. Biochem. 35, 669-677

Phan AT, Goldrath AW, Glass CK (2017): Metabolic and epigenetic coordination of T-cell and macrophage immunity. Immunity 46, 714-729

https://doi.org/10.1016/j.immuni.2017.04.016

Piskarev IM (2016): Active factors of low ionised plasma radiation produced in air spark discharge. RJPBCS 4, 1171-1189

Piskarev IM, Burkhina OE, Ivanova IP (2016a): Redox processes induced in methemoglobin solution by mercury lamp UV light and spark-discharge plasma radiation. High Energy Chemistry 50, 71-76

Piskarev IM, Ivanova IP, Samodelkin AG, Ivashenko MN (2016b): Initiation and Investigation of Free Radical Processes in Biological Experiments. FSBEI HE Nizhny Novgorod SAA, Nizhny Novgorod

Roca-Cusachs P, Almendros I, Sunyer R (2006): Rheology of passive and adhesion-activated neutrophils probed by atomic force microscopy. Biophysical Journal 91, 3508-3518 https://doi.org/10.1529/biophysj.106.088831

Salmin VV, Skomorokha DP, Reushev MY, Frolova OV, Malinovskaya NA, Lopatina OL, Salmina AB (2014): The effects of ultraviolet laser radiation on macrophages functional activity. Siberian medical review 4, 39-44 https://doi.org/10.20333/25000136-2014-4-39-44

Sanchez-Madrid F, del Pozo MA (1999): Leukocyte polarization in cell migration and immune interactions. EMBO J. 18, 501-511 https://doi.org/10.1093/emboj/18.3.501

Sasai Y, Kondo S, Yamauchi Y, Kuzuya M (2010): Plasma surface modification of polymer substrate for cell adhesion control. J. Photopolym. Sci. Technol. 23, 595-598

https://doi.org/10.2494/photopolymer.23.595

Seyrantepe V, Iannello A, Liang F, Kanshin E, Jayanth P, Samarani S, Szewczuk M, Ahmad A, Pshezhetsky AV (2010): Regulation of phagocytosis in macrophages by neuraminidase 1. J. Biol. Chem. 285, 206-215 https://doi.org/10.1074/jbc.M109.055475

Skowronska M, Albrecht J (2013): Oxidative and nitrosative stress in ammonia neurotoxicity. Neurochem. Int. 62, 731-737 https://doi.org/10.1016/j.neuint.2012.10.013

Varki A, Gagneux P (2012): Multifarious roles of sialic acids in immunity. Ann. N.Y. Acad. Sci. 1253, 16-36 https://doi.org/10.1111/j.1749-6632.2012.06517.x

Wang D, Ozhegov E, Wang L, Zhou A, Nie H, Li Y, Sun X (2016): Sialylation and desialylation dynamics of monocytesupon differentiation and polarization to macrophages. Glycoconj. J. 33, 725-733 
https://doi.org/10.1007/s10719-016-9664-4

Witko-Sarsat V, Friedlander M, Capeillère-Blandin C, NguyenKhoa T, Nguyen AT, Zingraff J, Jungers P, Descamps-Latscha B (1996): Advanced oxidation protein products as novel markers of oxidative stress in ischemia. Kidney International 49, 1304-1313

https://doi.org/10.1038/ki.1996.186
Yim MB, Kang SO, Chock PB (2000). Enzyme-like activity of glycated cross-linked proteins in free radical generation. Ann. N.Y. Acad. Sci. 899, 168-181

https://doi.org/10.1111/j.1749-6632.2000.tb06185.x

Received: March 30, 20018

Final version accepted: July 6, 2018

First published online: November 15, 2018 